\title{
Educación virtual, ¿un complemento para educación de pregrado en salud durante la pandemia?
}

\author{
Virtual education, a complement to undergraduate studies in health \\ education during the pandemic?
}

Winston Maldonado-Gómez 1,a

En la Resolución de Consejo Directivo de la Superintendencia Nacional de Educación Superior número 039-2020-Sunedu-CD(1), organismo público encargado de la regulación de la educación superior en el país, se norma el proceso de adaptación de la educación superior a la modalidad virtual. Esta resolución generó el debate en las facultades de medicina si la educación médica en pregrado podría adaptarse o no a la modalidad virtual.

La educación virtual a pesar de las limitaciones que presenta para la educación en salud, tiene varios beneficios como la necesidad de una comunicación más estrecha a través del aula virtual para compensar la falta de interacción presencial docente alumno; el desarrollo de una enseñanza por competencias, en la cual la forma principal de evaluación es la presentación de evidencias de los resultados de aprendizaje planificados al inicio del curso; y la posibilidad de utilizar pacientes simulados o casos clínicos estandarizados para el estudio de patología importantes por su pronóstico pero poco frecuentes en la práctica clínica.

Para el establecimiento de una educación por competencias propio de la educación virtual, el reconocimiento de las competencias que un profesional de la salud debe alcanzar es un punto de reflexión importante y necesario para la formación del futuro profesional en ciencias de la salud en general y la educación médica en particular. Este profesional no solamente debe conocer aspectos técnicos propios de la profesión, sino desarrollar actitudes, razonamiento ético; por tanto, es necesaria una visión holística de la formación profesional ${ }^{(2)}$.

El proceso de adaptación a la educación virtual requiere una planificación exhaustiva del proceso pedagógico, así como la capacitación de los docentes para que adquieran las competencias digitales necesarias para un adecuado desempeño y una mayor rigurosidad en todo el desarrollo de los procesos de enseñanza y aprendizaje ${ }^{(3)}$. Asimismo, la dedicación que un docente universitario debe realizar en la modalidad virtual es mayor, pues se deben elaborar y buscar los elementos necesarios para complementar los contenidos, estrategias, actividades y recursos utilizados durante las sesiones sincrónicas (en tiempo real).

La educación virtual, además de las estrategias educativas planteadas, requiere de aplicar estrategias enfocadas en fortalecer el desempeño en escenarios de desempeño profesional reales como el aprendizaje basado en problemas (ABP) y la evaluación del desempeño de los estudiantes en condiciones cercanas a lo real como en la evaluación clínica objetivo estructurada (ECOE). Por tanto, la educación virtual al enfocarse en los desempeños, establece en forma clara las competencias que el estudiante debe alcanzar, y dirige la selección de estrategias educativas y de contenidos que permitan cumplir con ese propósito, siendo lo más importante en esta modalidad de enseñanza el logro de los desempeños profesionales y no la cantidad de contenidos adquiridos.

Una de las principales deficiencias en la educación de pregrado es la ausencia de la investigación formativa, es decir la formación a través de las actividades de aprendizaje de competencias relacionadas a la investigación científica, como la redacción científica, la citación de referencias bibliográficas, la búsqueda de información científica en forma exhaustiva y la lectura crítica de la investigación científica, necesaria para una formación integral que le brinde la base científica al profesional de la salud ${ }^{(4)}$; que pueden

\footnotetext{
1. Facultad de Medicina Humana, Universidad Nacional Pedro Ruiz Gallo. Hospital Regional de Lambayeque. Lambayeque, Perú.

a. Magister en Medicina, Especialista en dermatología.
} 
ser adaptados totalmente a la modalidad virtual, están los de investigación científica en educación médica y ciencias de la salud, por ejemplo, las clases pueden ser realizadas en forma virtual y el seguimiento de los alumnos a través de asesorías en sesiones virtuales y correo electrónico; asimismo, se pueden hacer uso de las aulas virtuales para colocar a disposición de los alumnos lecturas y videos complementarios.

Nuestro país requiere un incremento significativo en la investigación en salud, pues aún no tenemos la cantidad y calidad necesaria para plantear alternativas de solución a nuestro contexto; por ejemplo, en la actual pandemia la generación de proyectos de investigación científica relacionada a ésta ha sido escasa. El desarrollo de la enseñanza virtual de la investigación no solo a nivel de las universidades, sino en los establecimientos de salud es una alternativa fundamental para fomentar la investigación científica en salud.

Considerando que la duración de la pandemia aún es una incertidumbre y que el escenario posterior a esta será diferente, esta realidad nos reta a buscar alternativas para la adaptación en la educación médica y en salud en general, ya que pueden encontrar en la educación virtual un complemento interesante a explorar durante y después de la crisis sanitaria, que permita potenciar todas las posibilidades de la educación presencial y la capacitación continua tanto en la formación en pregrado como en investigación científica en salud.

Financiamiento: Autofinanciado.

Conflicto de interés: El autor declara no tener conflictos de interés.

\section{REFERENCIAS BIBLIOGRÁFICAS}

1. Resolución del Consejo Directivo $\mathrm{N}^{\circ}$ 039-2020-SUNEDU/CD [Internet]. SUNEDU. 2020 [citado 17 de agosto de 2020]. Disponible en: https:// www.sunedu.gob.pe/resolucion-del-consejo-directivo-n-039-2020-sunedu-cd/

2. Millás-Mur J. Ética y bioética en el pregrado de medicina: una propuesta. Rev Peru Med Exp Salud Publica. enero de 2019;36(1):93-9.

3. Dreizzen E, Zangara A, Spinelli O. Formación de docentes de la carrera de Medicina en el diseño de contenidos educativos mediante Moodle. Rev Iberoam Tecnol En Educ Educ En Tecnol. junio de 2014;(13):72-9.

4. Rodríguez Abrahantes TN, Rodríguez Abrahantes A, García Pérez M. La investigación y su contribución formativa en estudiantes de las ciencias médicas. EDUMECENTRO. marzo de 2016;8(1):143-58. 